\title{
Untangling a Polygon
}

\author{
János Pach and Gábor Tardos \\ Rényi Institute of Mathematics \\ Hungarian Academy of Sciences \\ pach@cims.nyu.edu, tardos@renyi.hu
}

\begin{abstract}
The following problem was raised by M. Watanabe. Let $P$ be a self-intersecting closed polygon with $n$ vertices in general position. How manys steps does it take to untangle $P$, i.e., to turn it into a simple polygon, if in each step we can arbitrarily relocate one of its vertices. It is shown that in some cases one has to move all but at most $O\left((n \log n)^{2 / 3}\right)$ vertices. On the other hand, every polygon $P$ can be untangled in at most $n-\Omega(\sqrt{n})$ steps. Some related questions are also considered.
\end{abstract}

\section{Introduction}

Suppose we have a self-intersecting closed polygon $P$ on the screen of our computer, whose vertices are $p_{1}, p_{2}, \ldots, p_{n}$ in this order, and no three vertices are collinear. We are allowed to modify $P$ so that in each step we can grab a vertex and move it to an arbitrary new position. (For simplicity, we assume that the screen is very large, so we are not limited by its size.) At the 5th Czech-Slovak Symposium on Combinatorics in Prague in 1998, Mamoru Watanabe asked the following question. Is it true that every polygon $P$ can be untangled, i.e., turned into a noncrossing polygon, in at most $\varepsilon n$ steps, for some absolute constant $\varepsilon<1$ ?

The aim of this note is to answer this question in the negative.

Given another closed polygon $Q$ with vertices $q_{1}, q_{2}, \ldots, q_{n}$ (in this order), let $f(P, Q)$ denote the number of "common points" of $P$ and $Q$, i.e., the number of indices $i$, for which $q_{i}=p_{i}$. Let $f(P)$ denote the largest number of points that can be kept fixed when we untangle $P$. Using our notation,

$$
f(P)=\max _{Q} f(P, Q)
$$

where the maximum is taken over all noncrossing closed polygons with $n$ vertices. See Fig. 1.

It is easy to see that every polygon $P$ can be untangled in at most $n-\sqrt{n}$ moves. That is, we have

Proposition 1. For every polygon $P$ with $n$ vertices, we have $f(P)>\sqrt{n}$.

Proof. Assume without loss of generality that $p_{n}$ is a vertex of the convex hull of $\left\{p_{1}, p_{2}, \ldots, p_{n}\right\}$, and let $p_{\sigma(1)}, p_{\sigma(2)}, \ldots, p_{\sigma(n-1)}$ be the other points of $P$, listed in clockwise order of visibility around $p_{n}$. According to a wellknown lemma of Erdős

P. Mutzel, M. Jünger, and S. Leipert (Eds.): GD 2001, LNCS 2265, pp. 154-161, 2002.

(C) Springer-Verlag Berlin Heidelberg 2002 


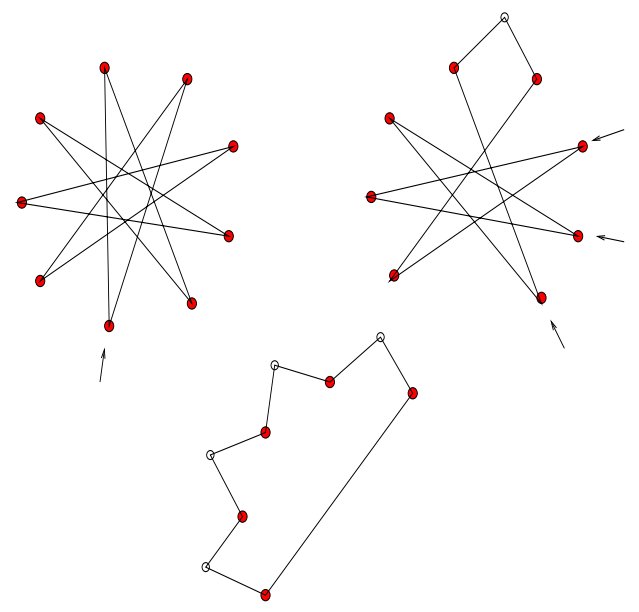

Fig. 1. For a star-polygon $P$ with $n$ vertices, we have $f(P)=\frac{n+1}{2}$

and Szekeres [ES35], every sequence of length $k$ has a monotone subsequence of length $\lceil\sqrt{k}\rceil$. Therefore, there is a sequence $1 \leq i_{1}<i_{2}<i_{3}<\ldots \leq n-1$ of length $\lceil\sqrt{n-1}\rceil$ such that either $\sigma\left(i_{1}\right)<\sigma\left(i_{2}\right)<\sigma\left(i_{3}\right)<\ldots$ or $\sigma\left(i_{1}\right)>$ $\sigma\left(i_{2}\right)>\sigma\left(i_{3}\right)>\ldots$ is true. In either case, the points $p_{n}, p_{\sigma\left(i_{1}\right)}, p_{\sigma\left(i_{2}\right)}, \ldots$ induce a noncrossing closed polygon $Q_{0}$. Let $Q$ denote the $n$-gon obtained from $Q_{0}$ by subdividing its sides with as many points as necessary, to achieve that the index of every point $p_{n}, p_{\sigma\left(i_{1}\right)}, p_{\sigma\left(i_{2}\right)}, \ldots$ be the same in $P$ as in $Q$. Clearly, we have $f(P, Q) \geq\lceil\sqrt{n-1}\rceil+1>\sqrt{n}$, as required.

Our main result can now be formulated as follows.

Theorem 2. For every sufficiently large n, there exists a closed polygon $P$ with $n$ vertices, which cannot be untangled in fewer than $n-c(n \log n)^{2 / 3}$ moves. That is, we have $f(P) \leq c(n \log n)^{2 / 3}$, where $c$ is a constant.

Let $G$ be a graph with vertex set $V(G)$ and edge set $E(G)$, respectively. A drawing of $G$ is a representation of $G$ in the plane such that every vertex corresponds to a point, and every edge is represented by a Jordan arc connecting the corresponding two points without passing through any vertex other than its endpoints. Two edges are said to cross each other if they have an interior point in common. The crossing number $\operatorname{cr}(G)$ of $G$ is defined as the minimum number of crossing pairs of arcs in a drawing of $G$.

For any partition of the vertex set of $G$ into two disjoint parts, $V_{1}$ and $V_{2}$, let $E\left(V_{1}, V_{2}\right) \subseteq E(G)$ denote the set of edges with one endpoint in $V_{1}$ and the other in $V_{2}$. Define the bisection width of $G$ as

$$
b(G)=\min \left|E\left(V_{1}, V_{2}\right)\right|,
$$


where the minimum is taken over all partitions $V(G)=V_{1} \cup V_{2}$ such that $\left|V_{1}\right|,\left|V_{2}\right| \leq 2 n / 3$.

Theorem 2 is established by a random construction. The proof is based on the following consequence of a weighted version of the Lipton-Tarjan separator theorem for planar graphs.

Lemma 3. [PSS94, [SV94] Let $G$ be a graph of $n$ vertices with degrees $d_{1}, d_{2}, \ldots$ $\ldots, d_{n}$. Then

$$
b^{2}(G) \leq(1.58)^{2}\left(16 \mathrm{cr}(G)+\sum_{k=1}^{n} d_{k}^{2}\right)
$$

where $b(G)$ and $\operatorname{cr}(G)$ denote the bisection width and the crossing number of $G$, respectively.

Corollary 4. Let $G$ be a graph of $n$ vertices with degrees $d_{1}, d_{2}, \ldots, d_{n}$. Then, for any edge disjoint subgraphs $G_{1}, G_{2}, \ldots, G_{j} \subseteq G$, we have

$$
\sum_{i=1}^{j} b\left(G_{i}\right) \leq 1.58 j^{1 / 2}\left(16 \mathrm{cr}(G)+\sum_{k=1}^{n} d_{k}^{2}\right)^{1 / 2} .
$$

Let $d_{i k}$ denote the degree of the $k$-th vertex in $G_{i}$. Corollary 4 immediately follows from Lemma 3. Indeed, applying Lemma 3 to each $G_{i}$ separately and adding up the resulting inequalities, we obtain

$$
\begin{aligned}
\sum_{i=1}^{j} b^{2}\left(G_{i}\right) & \leq(1.58)^{2}\left(16 \sum_{i=1}^{j} \operatorname{cr}\left(G_{i}\right)+\sum_{i=1}^{j} \sum_{k=1}^{n} d_{i k}^{2}\right) \\
& \leq(1.58)^{2}\left(16 \mathrm{cr}(G)+\sum_{k=1}^{n} d_{k}^{2}\right) .
\end{aligned}
$$

Therefore, we have

$$
\left(\sum_{i=1}^{j} b\left(G_{i}\right)\right)^{2} \leq j \sum_{i=1}^{j} b^{2}\left(G_{i}\right) \leq(1.58)^{2} j\left(16 \mathrm{cr}(G)+\sum_{k=1}^{n} d_{k}^{2}\right),
$$

as required.

\section{Proof of Theorem 2}

We start with an auxiliary lemma.

Lemma 5. Let $H_{b}$ denote the graph (cycle) defined on the vertex set $V=$ $\{1,2, \ldots, t\}$, whose edges are $(1,2),(2,3), \ldots,(t, 1)$. Let $H_{r}$ be a randomly selected Hamilton cycle on the same vertex set, i.e., let

$$
E\left(H_{r}\right)=\{(\sigma(1), \sigma(2)),(\sigma(2), \sigma(3)), \ldots,(\sigma(t), \sigma(1))\},
$$

where $\sigma$ is a random permutation of $V$. 
Then, for every $s<t$ and $K$, the probability that the crossing number of $H=H_{b} \cup H_{r}$ is at most $K$ satisfies

$$
\operatorname{Prob}[\operatorname{cr}(H) \leq K] \leq\left(\begin{array}{c}
t \\
D
\end{array}\right)^{2}\left(\frac{3 t}{s}\right)^{D} \frac{s^{t-D}}{(t-D) !},
$$

where $D=\lfloor 71 \sqrt{t(K+t) / s}\rfloor$.

Proof. We refer to the edges of $H_{b}$ and $H_{r}$ as black and red edges, respectively. Suppose that $\operatorname{cr}(H) \leq K$. The degree of every vertex in $H$ is at most 4 , so it follows from Corollary 4 that the sum of the bisection widths of any $j$ pairwise (edge) disjoint subgraphs of $H$ is at most

$$
2 \cdot 1.58 j^{1 / 2}(16 K+16 t)^{1 / 2}<13 j^{1 / 2}(K+t)^{1 / 2} .
$$

We multiplied the upper bound given in Corollary 4 by a factor of 2 , because any two vertices of $H$ may be connected by 2 edges: one black and one red.

Let $s$ be a positive integer. By deleting relatively few edges, decompose $H$ into connected components of sizes smaller than $s$ as follows. In the first step, delete $b(H)$ edges such that $H$ falls into two components, each having at most $\frac{2}{3}|V(H)|=\frac{2}{3} t$ vertices. As long as there is a component $H^{\prime} \subset H$ whose size is at least $s$, by the removal of $b\left(H^{\prime}\right)$ edges, cut it into two smaller components, each of size at most $(2 / 3)\left|V\left(H^{\prime}\right)\right|$. When there are no such components left, stop.

Let $\mathcal{H}$ denote the family of all components arising at any level of the above procedure (e.g., we have $H \in \mathcal{H}$ ), and let $\mathcal{H}_{0} \subseteq \mathcal{H}$ consist of the components of the final decomposition. That is, $\mathcal{H}_{0}$ contains all elements of $\mathcal{H}$, whose sizes are smaller than $s$. On the other hand, the size of every element of $\mathcal{H}_{0}$ is at least $s / 3$, so we have that

$$
\left|\mathcal{H}_{0}\right| \leq \frac{t}{s / 3}=\frac{3 t}{s},
$$

and the total number of cuts is $\left|\mathcal{H}_{0}\right|-1<3 t / s$.

Define the order of any element $H^{\prime} \in \mathcal{H}$ as the largest integer $k$, for which there is a chain

$$
H_{0} \nRightarrow H_{1} \nRightarrow \ldots \stackrel{\subsetneq}{\ddagger} H_{k}
$$

in $\mathcal{H}$ such that $H_{0} \in \mathcal{H}_{0}$ and $H_{k}=H^{\prime}$. Thus, $\mathcal{H}_{0}$ is the set of elements of $\mathcal{H}$ of order 0 . For any $k$, let $\mathcal{H}_{k}$ denote the set of all elements of $\mathcal{H}$ of order $k$.

For a fixed $k \geq 1$, the elements of $\mathcal{H}_{k}$ are pairwise (vertex) disjoint. Recall that in a chain (3) we have $\left|V\left(H_{1}\right)\right| \geq s$ and the ratio of the sizes of any two consecutive members is at least $3 / 2$. Therefore, the number of vertices in any element of $\mathcal{H}_{k}$ is at least $(3 / 2)^{k-1} s$, which in turn implies that for $k \geq 1$

$$
j_{k}:=\left|\mathcal{H}_{k}\right| \leq \frac{t}{(3 / 2)^{k-1} s}=\frac{(2 / 3)^{k-1} t}{s} .
$$

Applying (1) to the subgraphs (components) in $\mathcal{H}_{k}$, we obtain that the total number of edges removed, when they are first subdivided during our procedure, is at most

$$
13\left(\frac{2}{3}\right)^{\frac{k-1}{2}}\left(\frac{t}{s}\right)^{1 / 2}(K+t)^{1 / 2} .
$$


Summing up over all $k$, we conclude that the total number of edges deleted during the whole procedure does not exceed

$$
D=\left\lfloor 71 \sqrt{\frac{t(K+t)}{s}}\right\rfloor .
$$

Consequently, if we want to give an upper bound on $\operatorname{Prob}[\operatorname{cr}(H) \leq K]$, it is sufficient to bound the probability that $H$ can be decomposed into sets of size smaller than $s$ by the deletion of precisely $D$ black and precisely $D$ red edges. In what follows, we estimate this probability.

The $D$ black edges that are deleted can be chosen in $\left(\begin{array}{l}t \\ D\end{array}\right)$ different ways. The remaining black edges form $D$ paths. The vertex set of any subgraph in $\mathcal{H}$ is the union of the vertex sets of a few of these paths. By (2), there are at most $(3 t / s)^{D}$ possibilities for the partition of $V$ induced by $\mathcal{H}$, once the deleted black edges are chosen.

We consider the red Hamiltonian cycle to be picked with an orientation. There are $\left(\begin{array}{l}t \\ D\end{array}\right)$ different ways how to pick the starting points of the $D$ deleted red edges.

The probability that a randomly selected red Hamiltonian cycle "respects" a fixed partition of $V$ into parts of size smaller than $s$, except for the edges originating at a fixed set of size $D$, is at most $s^{t-D} /(t-D) !$. Indeed, when we start drawing $H_{r}$ randomly at a point, and we reach a vertex $x$ which is not the starting point of a deleted red edge, then the probability that the endpoint of the red edge starting at this point belongs to the part of the partition which contains $x$ is less than $s$ divided by the number of vertices in $V$ not yet visited by the initial portion of $H_{r}$. Summarizing: the probability that $H$ can be decomposed into sets of size smaller than $s$ by the deletion of $D$ black and $D$ red edges is at most

$$
\left(\begin{array}{c}
t \\
D
\end{array}\right)^{2}\left(\frac{3 t}{s}\right)^{D} \frac{s^{t-D}}{(t-D) !}
$$

and the lemma follows.

Now we are in a position to establish Theorem 2.

Consider a regular $n$-gon and let $p_{1}, p_{2}, \ldots, p_{n}$ be a random permutation of its vertices. Let $P$ denote the closed polygon obtained by connecting the $p_{i}$-s in this order. We claim that with high probability $f(P) \leq c(n \log n)^{2 / 3}$, where $c$ is a constant.

For any positive integer $t$, we have $f(P) \geq t$ if and only if there is a $t$-element subset $T \subseteq\left\{p_{1}, p_{2}, \ldots, p_{n}\right\}$ such that there is a noncrossing closed polygon $Q$ with vertices $q_{1}, q_{2}, \ldots, q_{n}$, in this order, with $q_{i}=p_{i}$ whenever $p_{i} \in T$.

To estimate the probability of this event for a fixed $t$-element set $T$, define two Hamilton cycles, $H_{b}$ and $H_{r}$, on the vertex set $T$ as follows. Let $H_{b}$ consist of all edges of the convex hull of $T$. These edges are called black. A vertex $p_{i} \in T$ is connected to another vertex $p_{j} \in T$ by an edge of $H_{r}$, if $p_{i}$ and $p_{j}$ are consequtive in the cyclic order induced on $T$ by the random permutation. That is, if $i<j$, then there is no index $k$ with $i<k<j$ such that and $p_{k} \in T$ or there is no 
index $k$ with $k<i$ or $j<k$ with $p_{k} \in T$. The edges in $H_{r}$ are said to be red. Let $H=H_{b} \cup H_{r}$.

Suppose now that there is a noncrossing closed polygon $Q$ with vertices $q_{1}, q_{2}, \ldots, q_{n}$, such $q_{i}=p_{i}$ whenever $p_{i} \in T$. By slightly changing the positions of its vertices not belonging to $T$, if necessary, we may achieve that the no three vertices of $Q$ are collinear.

Consider the drawing of $H$, in which every vertex is represented by itself, every black edge is represented by a straight line segment, and every red edge by the corresponding portion of $Q$. In this drawing, there is no crossing between edges of the same color. Since every edge of $Q$ can cross the black cycle (the boundary of the convex hull of $T$ ) in at most two points, we obtain the the number of crossings, and hence $\operatorname{cr}(H)$, are at most $2 n$. Thus, we have

$$
\operatorname{Prob}[f(P) \geq t] \leq\left(\begin{array}{l}
n \\
t
\end{array}\right) \operatorname{Prob}[\operatorname{cr}(H) \leq 2 n] .
$$

Notice that any fixed set $T$ uniquely determines $H_{b}$, but $H_{r}$ is a uniformly distributed random Hamiltonian cycle on $T$ determined by the random permutation $p_{1}, \ldots, p_{n}$. After substituting $t=150(n \log n)^{2 / 3}$ and applying Lemma 5 with $K=2 n$ and $s=101 n^{1 / 3} \log ^{4 / 3} n$, Theorem 2 follows by computation:

$$
\operatorname{Prob}[f(P) \geq t] \leq\left(\begin{array}{c}
n \\
t
\end{array}\right)\left(\begin{array}{c}
t \\
D
\end{array}\right)^{2}\left(\frac{3 t}{s}\right)^{D} \frac{s^{t-D}}{(t-D) !}
$$

Here $D=\lfloor 71 \sqrt{t(K+t) / s}\rfloor<t / \log n$ and hence we get

$$
\operatorname{Prob}[f(P) \geq t] \leq 2^{-t}
$$

\section{Related Problems and Remarks}

1. Proposition 1 (with a weaker constant) also follows from the main result in [PW98]: Every planar graph with $m$ vertices admits a crossing-free drawing in the plane such that its vertices are mapped into arbitrarily prespecified points and each of its edges are represented by a polygonal curve with fewer than $C m$ bends, where $C$ is a constant. (Apply this result to the cycle with vertices $p_{\lceil\sqrt{C n}\rceil}, p_{\lceil 2 \sqrt{C n}\rceil}, p_{\lceil 3 \sqrt{C n}\rceil}, \ldots$, where each of these points has to be mapped into itself.)

2. It is easy to see that any polygon of $n$ vertices and only one crossing pair of edges can be untangled in $\lceil n / 4\rceil$ moves. Indeed, deleting the two crossing edges, the polygon falls into two disjoint paths. Let $p_{1}, \ldots, p_{m}, m \leq n / 2$ denote the vertices of one of these paths in their natural order. We move $p_{1}$ close to the crossing of the two deleted edges and we move $p_{i}$ close to $p_{m+2-i}$ for $i=$ $2, \ldots,\lceil m / 2\rceil$. One can do this in a way to obtain a simple polypon. Figure 2 shows an example of a polygon with a single crossing that cannot be untangled with $o(n)$ moves and it seems that one cannot untangle it moving substantially fewer than $n / 4$ vertices. 


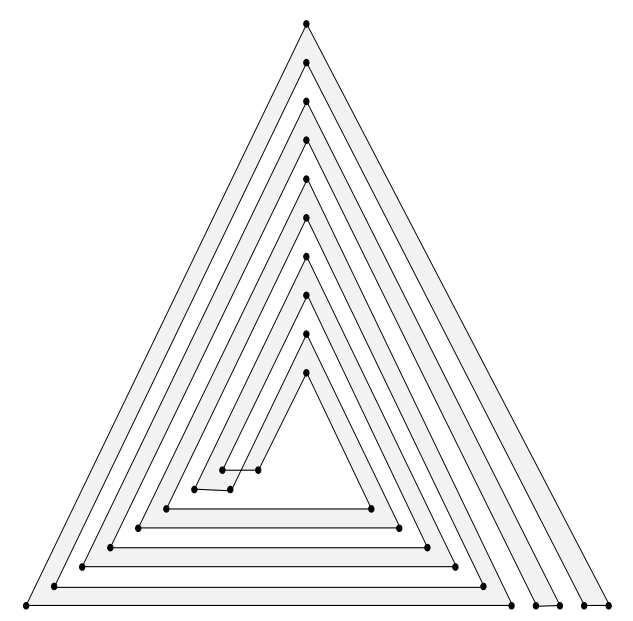

Fig. 2. A polygon with one crossing which cannot be untangled in few moves.

Obviously, if a polygon has $c$ crossings, then it can be untangled without moving the vertices of its longest crossing-free section, whose length is at least $\lceil n /(2 c)\rceil$. This bound is naturally far from being optimal.

More generally, we can raise the following

Problem 1. Let $P$ be a polygon of $n$ vertices with the property that every edge of $P$ crosses at most $k$ other edges. Is it true that $P$ can be untangled so that at least $c_{k} n$ vertices remain fixed, for a suitable constant $c_{k}>0$ depending only on $k$ ?

The answer to this question is in the affirmative in the special case when every edge $e$ of the polygon is disjoint from all other edges, whose distances from $e$ along $P$ are larger than a constant $k$.

3. One can ask similar questions for straight-line drawings of planar graphs rather than closed polygons. Now we are allowed to relocate any vertex, keeping all of its connections straight. Our goal is to get rid of all crossings, moving as few vertices as possible.

Problem 2. Let $P$ be a (not necessarily crossing-free) straight-line drawing of a planar graph with $n$ vertices. Can $P$ be untangled leaving $n^{\varepsilon}$ vertices fixed, for an absolute constant $\varepsilon>0$ ?

\section{References}

[ES35] P. Erdős and G. Szekeres, A combinatorial problem in geometry, Compositio Mathematica 2 (1935), 463-470.

[PSS94] J. Pach, F. Shahrokhi, and M. Szegedy, Applications of the crossing number, Proc. 10th ACM Symposium on Computational Geometry, 1994, 198-202. Also in: Algorithmica 16 (1996), 111-117. 
[PW98] J. Pach and R. Wenger, Embedding planar graphs with fixed vertex locations, in: Graph Drawing '98 (Sue Whitesides, ed.), Lecture Notes in Computer Science 1547, Springer-Verlag, Berlin, 1998, 263-274.

[SV94] O. Sýkora and I. Vrťo, On VLSI layouts of the star graph and related networks, Integration, The VLSI Journal 17 (1994), 83-93. 